\title{
Cyclic sum of multiple zeta values
}

\author{
by \\ YASUo OhNo and Noriko WakabaYashi (Osaka)
}

1. Introduction. In recent years, the multiple zeta values and multiple zeta-star values ("MZVs" and "MZSVs", respectively, for short) have appeared in various fields of mathematics and physics and attracted much interest (cf. [1], [3], [4], [12], [15]). At present, one of the central problems in this area is to clarify the structure of $\mathbb{Q}$-algebras generated by MZVs and MZSVs. (These algebras coincide with each other.) MZVs and MZSVs satisfy many relations, but their global structure is not yet fully understood. Here we will review their definitions and the well known basic identities, called the sum formulas for MZVs and MZSVs. The main purpose of this paper is to give a clean-cut decomposition of the sum formula for MZSVs and get a new family of relations between MZSVs and Riemann zeta values.

For any multi-index $\mathbf{k}=\left(k_{1}, \ldots, k_{n}\right)\left(k_{i} \in \mathbb{Z}, k_{i}>0\right)$, the weight $\mathrm{wt}(\mathbf{k})$, depth $\operatorname{dep}(\mathbf{k})$, and height $\mathrm{ht}(\mathbf{k})$ of $\mathbf{k}$ are by definition the integers $k=k_{1}+$ $\cdots+k_{n}, n$, and $s=\#\left\{i \mid k_{i}>1\right\}$, respectively. We denote by $I(k, n, s)$ the set of multi-indices $\mathbf{k}$ of weight $k$, depth $n$, and height $s$, and by $I_{0}(k, n, s)$ the subset of admissible indices, i.e., indices with the extra requirement that $k_{1} \geq 2$. We also use the set $I(k, n)=\bigcup_{s=1}^{\min (n, k-n)} I(k, n, s)$. For any admissible index $\mathbf{k}=\left(k_{1}, \ldots, k_{n}\right) \in I_{0}(k, n, s)$, the multiple harmonic series MZV $\zeta(\mathbf{k})$ and MZSV $\zeta^{*}(\mathbf{k})$ are defined by

$$
\begin{aligned}
& \zeta(\mathbf{k})=\zeta\left(k_{1}, \ldots, k_{n}\right)=\sum_{m_{1}>\cdots>m_{n}>0} \frac{1}{m_{1}^{k_{1}} \cdots m_{n}^{k_{n}}}, \\
& \zeta^{*}(\mathbf{k})=\zeta^{*}\left(k_{1}, \ldots, k_{n}\right)=\sum_{m_{1} \geq \cdots \geq m_{n} \geq 1} \frac{1}{m_{1}^{k_{1}} \cdots m_{n}^{k_{n}}} .
\end{aligned}
$$

Note that there exist natural linear relations between MZVs and MZSVs, for example,

2000 Mathematics Subject Classification: Primary 11M41.

The first author supported in part by JSPS Grant-in-Aid No. 15740025 and No. 15540190 and by Kinki University Grant No. 2003-GS02. 


$$
\begin{aligned}
\zeta^{*}\left(k_{1}, k_{2}\right)= & \zeta\left(k_{1}, k_{2}\right)+\zeta\left(k_{1}+k_{2}\right), \quad \zeta\left(k_{1}, k_{2}\right)=\zeta^{*}\left(k_{1}, k_{2}\right)-\zeta^{*}\left(k_{1}+k_{2}\right), \\
\zeta^{*}\left(k_{1}, k_{2}, k_{3}\right)= & \zeta\left(k_{1}, k_{2}, k_{3}\right)+\zeta\left(k_{1}+k_{2}, k_{3}\right)+\zeta\left(k_{1}, k_{2}+k_{3}\right) \\
& +\zeta\left(k_{1}+k_{2}+k_{3}\right), \\
\zeta\left(k_{1}, k_{2}, k_{3}\right)= & \zeta^{*}\left(k_{1}, k_{2}, k_{3}\right)-\zeta^{*}\left(k_{1}+k_{2}, k_{3}\right)-\zeta^{*}\left(k_{1}, k_{2}+k_{3}\right) \\
& +\zeta^{*}\left(k_{1}+k_{2}+k_{3}\right)
\end{aligned}
$$

and so the $\mathbb{Q}$-algebras of MZVs and MZSVs coincide with each other. Multiple zeta-star values $\zeta^{*}(\mathbf{k})$ were studied by Euler $([5])$, and his study is the origin of various investigations of multiple zeta values $\zeta(\mathbf{k})$.

The sum formulas were conjectured by C. Moen and M. E. Hoffman ([7]) and M. Schmidt and C. Markett ([10]) independently, and proved by A. Granville ([6]) and D. Zagier ([16]) independently. The statement of the formulas is as follows: For any integers $k>n>0$, we have

$$
\sum_{\left(k_{1}, k_{2}, \ldots, k_{n+1}\right) \in I(k, n+1)} \zeta\left(k_{1}+1, k_{2}, \ldots, k_{n+1}\right)=\zeta(k+1)
$$

and

$$
\sum_{\left(k_{1}, k_{2}, \ldots, k_{n+1}\right) \in I(k, n+1)} \zeta^{*}\left(k_{1}+1, k_{2}, \ldots, k_{n+1}\right)=\left(\begin{array}{c}
k \\
n
\end{array}\right) \zeta(k+1)
$$

(see also [8], [9], [11], [13], [14]).

In this article, we introduce a new classification of MZSVs to give a clean-cut decomposition of the sum formula. The definition is an analogy of Hoffman's cyclic equivalence classes for MZVs ([8]). However, the totals of the values in each class for the present case are much simpler than the ones for MZVs. They are integral multiples of a single Riemann zeta value. We shall state the result more precisely in the next section.

2. Cyclic equivalence classes of MZSVs and the main result. In this section we define the cyclic classes of MZSVs and state the main result. The proof is given in the next section, together with a new proof of the sum formula for MZSVs.

The cyclic sum formula for MZVs was conjectured by M. E. Hoffman and proved by the first author ([8]). Similarly we define cyclic equivalence classes of multiple indices in the set

$$
I(k, n)=\left\{\left(k_{1}, \ldots, k_{n}\right) \mid k_{1}+\cdots+k_{n}=k, k_{1}, \ldots, k_{n} \geq 1\right\} .
$$

We say two elements of $I(k, n)$ are cyclically equivalent if they are cyclic permutations of each other, i.e., for $\sigma=\left(k_{1}, \ldots, k_{n}\right)$ and $j=1, \ldots, n$, we define $\left(k_{1}, \ldots, k_{n}\right) \equiv\left(\sigma^{j}\left(k_{1}\right), \ldots, \sigma^{j}\left(k_{n}\right)\right)$. Let $\Pi(k, n)$ be the set of cyclic equivalence classes of $I(k, n)$. We denote by $|\alpha|$ the cardinality of the set $\alpha$. Then our main result is as follows: 
TheOREM 1. For any index set $\alpha \in \Pi(k, n)$ with $0<n<k$, we have

$$
\sum_{\left(k_{1}, k_{2}, \ldots, k_{n}\right) \in \alpha} \sum_{i=0}^{k_{1}-2} \zeta^{*}\left(k_{1}-i, k_{2}, \ldots, k_{n}, i+1\right)=\frac{k|\alpha|}{n} \zeta(k+1)
$$

where the inner sum on the left-hand side is treated as 0 whenever $k_{1}=1$.

In general, the cyclic sum formula for MZVs, given in [8], has linear combinations on both sides of the identity. The right-hand side of the above identity is an integral multiple of a single Riemann zeta value.

Here we give a table of classification of MZSVs of weights less than 8 .

\begin{tabular}{|c|c|c|c|c|c|}
\hline wt. & dep. & rep. of $\alpha$ & MZSVs & total & $n /|\alpha|$ \\
\hline 3 & 2 & $(2)$ & $\zeta^{*}(2,1)$ & $2 \zeta(3)$ & 1 \\
\hline \multirow[t]{2}{*}{4} & 2 & $(3)$ & $\zeta^{*}(3,1), \zeta^{*}(2,2)$ & $3 \zeta(4)$ & 1 \\
\hline & 3 & $(2,1)$ & $\zeta^{*}(2,1,1)$ & $3 \zeta(4)$ & 1 \\
\hline \multirow[t]{4}{*}{5} & 2 & $(4)$ & $\zeta^{*}(4,1), \zeta^{*}(3,2), \zeta^{*}(2,3)$ & $4 \zeta(5)$ & 1 \\
\hline & 3 & $(3,1)$ & $\zeta^{*}(3,1,1), \zeta^{*}(2,1,2)$ & $4 \zeta(5)$ & 1 \\
\hline & 3 & $(2,2)$ & $\zeta^{*}(2,2,1)$ & $2 \zeta(5)$ & 2 \\
\hline & 4 & $(2,1,1)$ & $\zeta^{*}(2,1,1,1)$ & $4 \zeta(5)$ & 1 \\
\hline \multirow[t]{6}{*}{6} & 2 & $(5)$ & $\zeta^{*}(5,1), \zeta^{*}(4,2), \zeta^{*}(3,3), \zeta^{*}(2,4)$ & $5 \zeta(6)$ & 1 \\
\hline & 3 & $(4,1)$ & $\zeta^{*}(4,1,1), \zeta^{*}(3,1,2), \zeta^{*}(2,1,3)$ & $5 \zeta(6)$ & 1 \\
\hline & 3 & $(3,2)$ & $\zeta^{*}(3,2,1), \zeta^{*}(2,2,2), \zeta^{*}(2,3,1)$ & $5 \zeta(6)$ & 1 \\
\hline & 4 & $(3,1,1)$ & $\zeta^{*}(3,1,1,1), \zeta^{*}(2,1,1,2)$ & $5 \zeta(6)$ & 1 \\
\hline & 4 & $(2,2,1)$ & $\zeta^{*}(2,2,1,1), \zeta^{*}(2,1,2,1)$ & $5 \zeta(6)$ & 1 \\
\hline & 5 & $(2,1,1,1)$ & $\zeta^{*}(2,1,1,1,1)$ & $5 \zeta(6)$ & 1 \\
\hline \multirow[t]{12}{*}{7} & 2 & (6) & $\zeta^{*}(6,1), \zeta^{*}(5,2), \zeta^{*}(4,3), \zeta^{*}(3,4), \zeta^{*}(2,5)$ & $6 \zeta(7)$ & 1 \\
\hline & 3 & $(5,1)$ & $\zeta^{*}(5,1,1), \zeta^{*}(4,1,2), \zeta^{*}(3,1,3), \zeta^{*}(2,1,4)$ & $6 \zeta(7)$ & 1 \\
\hline & 3 & $(4,2)$ & $\zeta^{*}(4,2,1), \zeta^{*}(3,2,2), \zeta^{*}(2,2,3), \zeta^{*}(2,4,1)$ & $6 \zeta(7)$ & 1 \\
\hline & 3 & $(3,3)$ & $\zeta^{*}(3,3,1), \zeta^{*}(2,3,2)$ & $3 \zeta(7)$ & 2 \\
\hline & 4 & $(4,1,1)$ & $\zeta^{*}(4,1,1,1), \zeta^{*}(3,1,1,2), \zeta^{*}(2,1,1,3)$ & $6 \zeta(7)$ & 1 \\
\hline & 4 & $(3,2,1)$ & $\zeta^{*}(3,2,1,1), \zeta^{*}(2,2,1,2), \zeta^{*}(2,1,3,1)$ & $6 \zeta(7)$ & 1 \\
\hline & 4 & $(3,1,2)$ & $\zeta^{*}(3,1,2,1), \zeta^{*}(2,1,2,2), \zeta^{*}(2,3,1,2)$ & $6 \zeta(7)$ & 1 \\
\hline & 4 & $(2,2,2)$ & $\zeta^{*}(2,2,2,1)$ & $2 \zeta(7)$ & 3 \\
\hline & 5 & $(3,1,1,1)$ & $\zeta^{*}(3,1,1,1,1), \zeta^{*}(2,1,1,1,2)$ & $6 \zeta(7)$ & 1 \\
\hline & 5 & $(2,2,1,1)$ & $\zeta^{*}(2,2,1,1,1), \zeta^{*}(2,1,1,2,1)$ & $6 \zeta(7)$ & 1 \\
\hline & 5 & $(2,1,2,1)$ & $\zeta^{*}(2,1,2,1,1)$ & $3 \zeta(7)$ & 2 \\
\hline & 6 & $(2,1,1,1,1)$ & $\zeta^{*}(2,1,1,1,1,1)$ & $6 \zeta(7)$ & 1 \\
\hline
\end{tabular}

Together with other known results (for example, by the formula in [2]), we get the following relations. 
ExAmples. (a) For any $n>1$, we have

$$
\sum_{i=0}^{n-2} \zeta^{*}(\underbrace{2, \ldots, 2}_{i}, 3, \underbrace{2, \ldots, 2}_{n-2-i}, 1)=\left(2^{2-2 n}+2 n-3\right) \zeta(2 n) .
$$

Indeed, we use Theorem 1 for $\alpha=\{(3, \underbrace{2, \ldots, 2}_{n-2})\}$, and replace $\zeta^{*}(\underbrace{2, \ldots, 2}_{n})$ by using $\zeta^{*}(\underbrace{2, \ldots, 2}_{n})=2\left(1-2^{1-2 n}\right) \zeta(2 n)$, which is a special case $(k=2 n=2 s)$ of T. Aoki and the first author's result ([2])

$$
\sum_{n=s}^{k-s} \sum_{\mathbf{k} \in I_{0}(k, n, s)} \zeta^{*}(\mathbf{k})=2\left(\begin{array}{c}
k-1 \\
2 s-1
\end{array}\right)\left(1-2^{1-k}\right) \zeta(k) .
$$

(b) For any positive integers $l$ and $m$, we have

$$
\zeta^{*}(\underbrace{2, \underbrace{1, \ldots, 1}_{m-1}}_{l m}, 2, \underbrace{1, \ldots, 1}_{m-1}, \ldots, 2, \underbrace{1, \ldots, 1}_{m-1}, 1)=(m+1) \zeta(l(m+1)+1) .
$$

For example, if we put $m=2$, we have

$$
\zeta^{*}(\underbrace{2,1,2,1, \ldots, 2,1}_{2 l}, 1)=3 \zeta(3 l+1) .
$$

3. Proof of Theorem 1 and the sum formula. After proving the main result, we will give an elementary proof of the sum formula by using Theorem 1.

In the proof of Theorem 1, we need a key fact on an infinite series $C$ defined as follows. For any positive integers $n, k_{1}, \ldots, k_{n}$ with $k_{1}+\cdots+k_{n}$ $>n$ (i.e., at least one of the $k_{i}$ 's is $>1$ ), define $C\left(k_{1}, \ldots, k_{n}\right)$ as the convergent series

$$
C\left(k_{1}, \ldots, k_{n}\right)=\sum_{\substack{a_{1} \geq \cdots \geq a_{n} \geq a_{n+1} \geq 1 \\ a_{1} \neq a_{n+1}}} \frac{1}{a_{1}^{k_{1}} \cdots a_{n}^{k_{n}}\left(a_{1}-a_{n+1}\right)} .
$$

Key Lemma 1. For any positive integers $n$ and $k_{1}, \ldots, k_{n}$ with $k_{i}>1$ for some $i$, we have

$$
\begin{aligned}
C\left(k_{1}, k_{2}, \ldots, k_{n}\right)- & C\left(k_{2}, k_{3}, \ldots, k_{n}, k_{1}\right) \\
& =k_{1} \zeta(k+1)-\sum_{i=0}^{k_{1}-2} \zeta^{*}\left(k_{1}-i, k_{2}, k_{3}, \ldots, k_{n}, i+1\right),
\end{aligned}
$$

where we put $k=k_{1}+\cdots+k_{n}$ and the sum on the right is understood to be 0 if $k_{1}=1$. 
Proof. For any non-negative integer $i \leq k_{1}-2$, we have

$$
\begin{aligned}
& \sum_{\substack{a_{1} \geq a_{2} \geq \cdots \geq a_{n} \geq a_{n+1} \geq 1 \\
a_{1} \neq a_{n+1}}} \frac{1}{a_{1}^{k_{1}-i} a_{2}^{k_{2}} \cdots a_{n}^{k_{n}} a_{n+1}^{i}\left(a_{1}-a_{n+1}\right)} \\
& =\sum_{a_{1} \geq a_{2} \geq \cdots \geq a_{n} \geq a_{n+1} \geq 1} \frac{1}{a_{1}^{k_{1}-i-1} a_{2}^{k_{2}} \cdots a_{n} k_{n} a_{n+1}^{i+1}}\left(\frac{1}{a_{1}-a_{n+1}}-\frac{1}{a_{1}}\right) \\
& =\sum_{\substack{a_{1} \geq a_{2} \geq \cdots \geq a_{n} \geq a_{n+1} \geq 1 \\
a_{1} \neq a_{n+1}}} \frac{1}{a_{1}^{k_{1}-i-1} a_{2}^{k_{2}} \cdots a_{n}^{k_{n}} a_{n+1}^{i+1}\left(a_{1}-a_{n+1}\right)} \\
& -\sum_{\substack{a_{1} \geq a_{2} \geq \cdots \geq a_{n} \geq a_{n+1} \geq 1 \\
a_{1} \neq a_{n+1}}} \frac{1}{a_{1}^{k_{1}-i} a_{2}^{k_{2}} \cdots a_{n}^{k_{n}} a_{n+1}^{i+1}} \\
& =\sum_{\substack{a_{1} \geq a_{2} \geq \cdots \geq a_{n} \geq a_{n+1} \geq 1 \\
a_{1} \neq a_{n+1}}} \frac{1}{a_{1}^{k_{1}-i-1} a_{2}^{k_{2}} \cdots a_{n}^{k_{n}} a_{n+1}^{i+1}\left(a_{1}-a_{n+1}\right)} \\
& -\sum_{a_{1} \geq a_{2} \geq \cdots \geq a_{n} \geq a_{n+1} \geq 1} \frac{1}{a_{1}^{k_{1}-i} a_{2}^{k_{2}} \cdots a_{n}^{k_{n}} a_{n+1}^{i+1}}+\sum_{a \geq 1} \frac{1}{a^{k_{1}-i+k_{2}+k_{3}+\cdots+k_{n}+i+1}} \\
& =\sum_{\substack{a_{1} \geq a_{2} \geq \cdots \geq a_{n} \geq a_{n+1} \geq 1 \\
a_{1} \neq a_{n+1}}} \frac{1}{a_{1}^{k_{1}-i-1} a_{2}^{k_{2}} \cdots a_{n}^{k_{n}} a_{n+1}^{i+1}\left(a_{1}-a_{n+1}\right)} \\
& -\zeta^{*}\left(k_{1}-i, k_{2}, \ldots, k_{n}, i+1\right)+\zeta(k+1) .
\end{aligned}
$$

Adding up the above equality for $i=0,1, \ldots, k_{1}-2$, we obtain

$$
\begin{aligned}
C\left(k_{1}, k_{2}, \ldots, k_{n}\right)= & \sum_{\substack{a_{1} \geq a_{2} \geq \cdots \geq a_{n} \geq a_{n+1} \geq 1 \\
a_{1} \neq a_{n+1}}} \frac{1}{a_{1} a_{2}^{k_{2}} \cdots a_{n}^{k_{n}} a_{n+1}^{k_{1}-1}\left(a_{1}-a_{n+1}\right)} \\
& -\sum_{i=0}^{k_{1}-2} \zeta^{*}\left(k_{1}-i, k_{2}, \ldots, k_{n}, i+1\right)+\left(k_{1}-1\right) \zeta(k+1) .
\end{aligned}
$$

The first sum on the right-hand side can be written as

$$
\begin{aligned}
\sum_{\substack{a_{1} \geq a_{2} \geq \cdots \geq a_{n+1} \geq 1 \\
a_{1} \neq a_{n+1}}} & \frac{1}{a_{2}^{k_{2}} \cdots a_{n}^{k_{n}} a_{n+1}^{k_{1}}}\left(\frac{1}{a_{1}-a_{n+1}}-\frac{1}{a_{1}}\right) \\
= & \sum_{\substack{a_{2} \geq a_{3} \geq \cdots \geq a_{n+1} \geq 1 \\
a_{2} \neq a_{n+1}}} \frac{1}{a_{2}^{k_{2}} \cdots a_{n}^{k_{n}} a_{n+1}^{k_{1}}} \sum_{a_{1} \geq a_{2}}\left(\frac{1}{a_{1}-a_{n+1}}-\frac{1}{a_{1}}\right) \\
& +\sum_{a_{2}=a_{3}=\cdots=a_{n+1} \geq 1} \frac{1}{a_{2}^{k_{2}} \cdots a_{n}^{k_{n}} a_{n+1}^{k_{1}}} \sum_{a_{1}>a_{2}}\left(\frac{1}{a_{1}-a_{n+1}}-\frac{1}{a_{1}}\right)
\end{aligned}
$$




$$
\begin{aligned}
& =\sum_{\substack{a_{2} \geq a_{3} \geq \cdots \geq a_{n+1} \geq 1 \\
a_{2} \neq a_{n+1}}} \frac{1}{a_{2}^{k_{2}} \cdots a_{n}^{k_{n}} a_{n+1}^{k_{1}}} \sum_{a_{n+2}=1}^{a_{n+1}} \frac{1}{a_{2}-a_{n+2}} \\
& +\sum_{a_{2}=a_{3}=\cdots=a_{n+1} \geq 1} \frac{1}{a_{2}^{k_{2}} \cdots a_{n}^{k_{n}} a_{n+1}^{k_{1}}} \sum_{a_{n+2}=0}^{a_{n+1}-1} \frac{1}{a_{2}-a_{n+2}} \\
& =\sum_{\substack{a_{2} \geq a_{3} \geq \cdots \geq a_{n+1} \geq 1 \\
a_{2} \neq a_{n+1}}} \frac{1}{a_{2}^{k_{2}} \cdots a_{n}^{k_{n}} a_{n+1}^{k_{1}}} \sum_{a_{n+2}=1}^{a_{n+1}} \frac{1}{a_{2}-a_{n+2}} \\
& +\sum_{a_{2}=a_{3}=\cdots=a_{n+1} \geq 1} \frac{1}{a_{2}^{k_{2}} \cdots a_{n}^{k_{n}} a_{n+1}^{k_{1}}} \sum_{a_{n+2}=1}^{a_{n+1}-1} \frac{1}{a_{2}-a_{n+2}}+\sum_{a \geq 1} \frac{1}{a^{k_{2}+\cdots+k_{n}+k_{1}+1}} \\
& =\sum_{\substack{a_{2} \geq a_{3} \geq \cdots \geq a_{n+2} \geq 1 \\
a_{2} \neq a_{n+2}}} \frac{1}{a_{2}^{k_{2}} \cdots a_{n}^{k_{n}} a_{n+1}^{k_{1}}\left(a_{2}-a_{n+2}\right)}+\sum_{a \geq 1} \frac{1}{a^{k+1}} \\
& =C\left(k_{2}, \ldots, k_{n}, k_{1}\right)+\zeta(k+1) \text {. }
\end{aligned}
$$

Thus we get the equality of Key Lemma 1.

Proof of Theorem 1. The formula is readily obtained when we apply the Key Lemma 1 for all cyclic permutations of $\left(k_{1}, \ldots, k_{n}\right) \in \alpha$ and add them up.

It is worth pointing out that Theorem 1 provides another proof of the sum formula.

Proof of the sum formula via Theorem 1. For each $\alpha \in \Pi(k, n)$, the number of MZSVs in the corresponding class is $(k-n)|\alpha| / n$, and so the mean value of each MZSVs can be computed as

$$
\frac{k|\alpha|}{n} \zeta(k+1) \cdot \frac{n}{(k-n)|\alpha|}=\frac{k}{k-n} \zeta(k+1),
$$

by using Theorem 1 . It is well known that the number of MZSVs with weight $k+1$ and depth $n+1$ is $\left(\begin{array}{c}k-1 \\ n\end{array}\right)$. So multiplying these values yields

$$
\frac{k}{k-n} \zeta(k+1) \cdot\left(\begin{array}{c}
k-1 \\
n
\end{array}\right)=\left(\begin{array}{l}
k \\
n
\end{array}\right) \zeta(k+1)
$$

as the sum of all MZSVs of weight $k+1$ and depth $n+1$, and finally we get the sum formula for MZSVs:

$$
\sum_{\left(k_{1}, k_{2}, \ldots, k_{n+1}\right) \in I(k, n+1)} \zeta^{*}\left(k_{1}+1, k_{2}, \ldots, k_{n+1}\right)=\left(\begin{array}{l}
k \\
n
\end{array}\right) \zeta(k+1) .
$$




\section{References}

[1] T. Aoki, S. Kanemitsu, M. Nakahara and Y. Ohno (eds.), Zeta Functions, Topology and Quantum Physics, Dev. Math. 14, Springer, 2005.

[2] T. Aoki and Y. Ohno, Sum relations for multiple zeta values and connection formulas for the Gauss hypergeometric functions, Publ. Res. Inst. Math. Sci. 41 (2005), 329337.

[3] J. M. Borwein, D. H. Bailey and R. Girgensohn, Experimentation in Mathematics, A. K. Peters, 2004.

[4] J. M. Borwein, D. M. Bradley, D. J. Broadhurst and P. Lisoněk, Special values of multiple polylogarithms, Trans. Amer. Math. Soc. 353 (2001), 907-941.

[5] L. Euler, Meditationes circa singulare serierum genus, Novi Comm. Acad. Sci. Petropol 20 (1775), 140-186, reprinted in: Opera Omnia, ser. I, Vol. 15, B. G. Teubner, Berlin, 1927, 217-267.

[6] A. Granville, A decomposition of Riemann's zeta-function, in: Analytic Number Theory, Y. Motohashi (ed.), London Math. Soc. Lecture Note Ser. 247, Cambridge, 1997, 95-101.

[7] M. Hoffman, Multiple harmonic series, Pacific J. Math. 152 (1992), 275-290.

[8] M. Hoffman and Y. Ohno, Relations of multiple zeta values and their algebraic expression, J. Algebra 262 (2003), 332-347.

[9] K. Ihara, M. Kaneko and D. Zagier, Derivation relations and regularized double shuffle relations of multiple zeta values, Compositio Math., to appear.

[10] C. Markett, Triple sums and the Riemann zeta function, J. Number Theory 48 (1994), 113-132.

[11] Y. Ohno, A generalization of the duality and sum formulas on the multiple zeta values, ibid. 74 (1999), 39-43.

[12] —, Sum relations for multiple zeta values, in: Zeta Functions, Topology and Quantum Physics, Dev. Math. 14, Springer, 2005, 131-144.

[13] Y. Ohno and D. Zagier, Multiple zeta values of fixed weight, depth, and height, Indag. Math. 12 (2001), 483-487.

[14] J. Okuda and K. Ueno, Relations for multiple zeta values and Mellin transforms of multiple polylogarithms, Publ. Res. Inst. Math. Sci. 40 (2004), 537-564.

[15] D. Zagier, Values of zeta functions and their applications, in: Proc. ECM 1992, Progr. Math. 120, 1994, 497-512.

[16] —, Multiple zeta values, unpublished manuscript, Bonn, 1995.

Department of Mathematics

Kinki University

Higashi-Osaka, Osaka 577-8502, Japan

E-mail: ohno@math.kindai.ac.jp

noriko@math.kindai.ac.jp

Received on 19.9.2005

and in revised form on 26.1.2006 